\title{
NOTE
}

\section{Oxygen isotopic behavior of precipitating silica from geothermal water}

\author{
ITSURO KITA ${ }^{1}$ and SACHIHIRO TAGUCHI ${ }^{2}$ \\ Research Institute of Natural Resources, Mining College, Akita University, \\ Akita 010,1* and Research Institute of Industrial Science, Kyushu \\ University, Kasuga, Fukuoka $816,{ }^{2}$ Japan
}

(Received December 23, 1985: Accepted May 14, 1986)

\begin{abstract}
Oxygen isotopic ratios were measured for the silica which was precipitated from the solution in process of evaporative concentration of Otake geothermal water at 76 and $88^{\circ} \mathrm{C}$. The oxygen isotopic ratios of the silica precipitates were extremely out of isotopic equilibrium with the solution in which the colloidal silica was observable. The fractionation factors for the "silica-solution in evaporative concentration system" were compared with those obtained for "the silica-flowing geothermal water system" where the silica is in oxygen isotopic equilibrium with the water. The difference is explained by isotopic zoning of the growing amorphous silica particles. The rearrangement and interlinkage of adsorbed silica on the surface of the amorphous silica contributes to attaining oxygen isotopic equilibrium between the silica precipitate and water. Amorphous silica phase once formed does not re-equilibrate with the solution at later stages. Hence the apparent oxygen isotopic fractionation between the bulk silica precipitate and the solution at the final stage seems to be out of equilibrium.
\end{abstract}

\section{INTRODUCTION}

Amorphous silica precipitated in the reinjection lines of geothermal power plants has been found to be in oxygen isotopic equilibrium with the flowing geothermal waters at a low temperature range of 34 to $93^{\circ} \mathrm{C}$ (Kita et al., 1985). In that case, the silica was precipitated from the waters of which isotopic ratios are constant during the precipitation. The temperature dependence of the fractionation factor was almost identical with that of the equilibrium fractionation reported for the quartz-water system (Matsuhisa et al., 1979). In the present study, we precipitated silica by concentrating evaporatively the geothermal water at constant temperatures, and measured the oxygen isotope fractionation factor between water and the precipitated silica. Under such conditions the oxygen isotopic ratio of the geothermal water changes gradually during concentration due to evaporation. We will discuss the oxygen isotopic behavior of silica precipitation under the two different conditions in view of the mechanism of the silica precipitation from geothermal water.

\section{EXPERIMENTAL}

Precipitation of amorphous silica from evaporating geothermal water

The geothermal water flowing at $93^{\circ} \mathrm{C}$ in a reinjection line of the Otake geothermal power plant, Kyushu, Japan, was used for this study. Just after collection, about $500 \mathrm{m \ell}$ of the geothermal water was kept at $76 \pm 2$ and $88 \pm$ $3^{\circ} \mathrm{C}$ with stirring, and concentrated evaporatively until colloidal silica was observable. Aliquots of the solution were sampled to measure the change in the $\delta^{18} \mathrm{O}$ value. The deposited silica was filtered from the solution, washed with distilled water and then air-dried at room

\footnotetext{
* Formerly Research Institute of Underground Resources, Akita University, (renamed in April, 1986).
} 
temperature.

\section{Isotopic analyses}

Oxygen isotope ratios of the silica samples were measured with a fluorination technique (Kita and Matsubaya, 1983) and those of the solutions with the conventional $\mathrm{H}_{2} \mathrm{O}-\mathrm{CO}_{2}$ equilibration method. The isotope ratios were expressed by $\delta^{18} \mathrm{O}$ values relative to the SMOW standard. The $\delta^{18} \mathrm{O}$ of silica was referred to SMOW on the basis of a fractionation factor of 1.0407 between $\mathrm{CO}_{2}$ and $\mathrm{H}_{2} \mathrm{O}$ (O'Neil and Epstein, 1966). The $\delta^{18} \mathrm{O}$ value of $\mathrm{NBS}-28$ reference quartz is $+9.36 \%$ with this method.

\section{Results AND Discussion}

Figure 1 shows the changes in the $\delta^{18} \mathrm{O}$ value of the water during evaporative concentration at 76 and $88^{\circ} \mathrm{C}$. Observable colloidal silica started to form from the solutions when the remaining volume decreased to 50 and $60 \mathrm{ml}$ (about $10 \%$ of the initial volume) at respective temperatures. The $\delta^{18} \mathrm{O}$ values of the silica precipitates were 28.9 and $25.0 \%$ at 76 and $88^{\circ} \mathrm{C}$, respectively, and those of the solutions were 23.8 and $11.2 \%$, respectively. The apparent fractionation factor between the silica precipitate and solution is defined as

$$
\begin{array}{r}
\alpha=\left(1+10^{-3} \delta^{18} \mathrm{O}_{\text {silica }}\right) / \\
\left(1+10^{-3} \delta^{18} \mathrm{O}_{\text {solution }}\right) .
\end{array}
$$

The obtained fractionation factors are 1.0050 and 1.0136 at 76 and $88^{\circ} \mathrm{C}$, respectively. As shown in Fig. 2, these values are extremely smaller than the equilibrium values reported in the previous experiments (Kita et al., 1985). Moreover, it is noted that the apparent fractionation factor is smaller at the lower tempera-

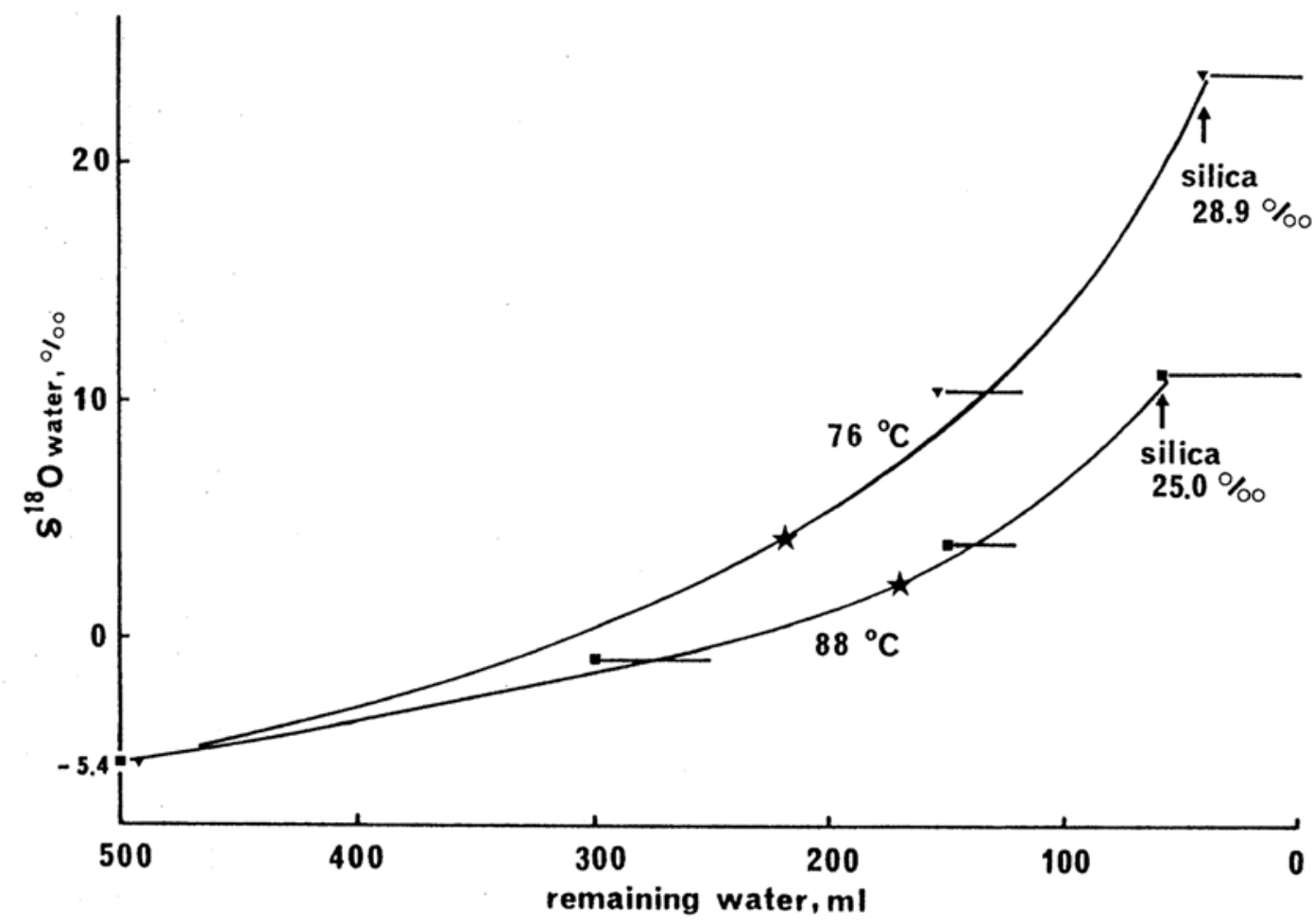

Fig. 1 Changes in the $\delta^{18} \mathrm{O}$ value of the solution during evaporative concentration at 76 and $88^{\circ} \mathrm{C}$. The horizontal bar shows the volume of the solution for which isotopic measurement was made. The $\delta^{18} \mathrm{O}$ values of silica represent those of the amorphous silica which were filtered from the solutions. The mark, $\star$, indicates the calculated $\delta^{18} \mathrm{O}$ values of the solutions which are in equilibrium with the deposited silica. 
ture of silica precipitation and that the smaller fractionation factor is accompanied by larger change in the $\delta^{18} \mathrm{O}$ value of solution (Fig. 1).

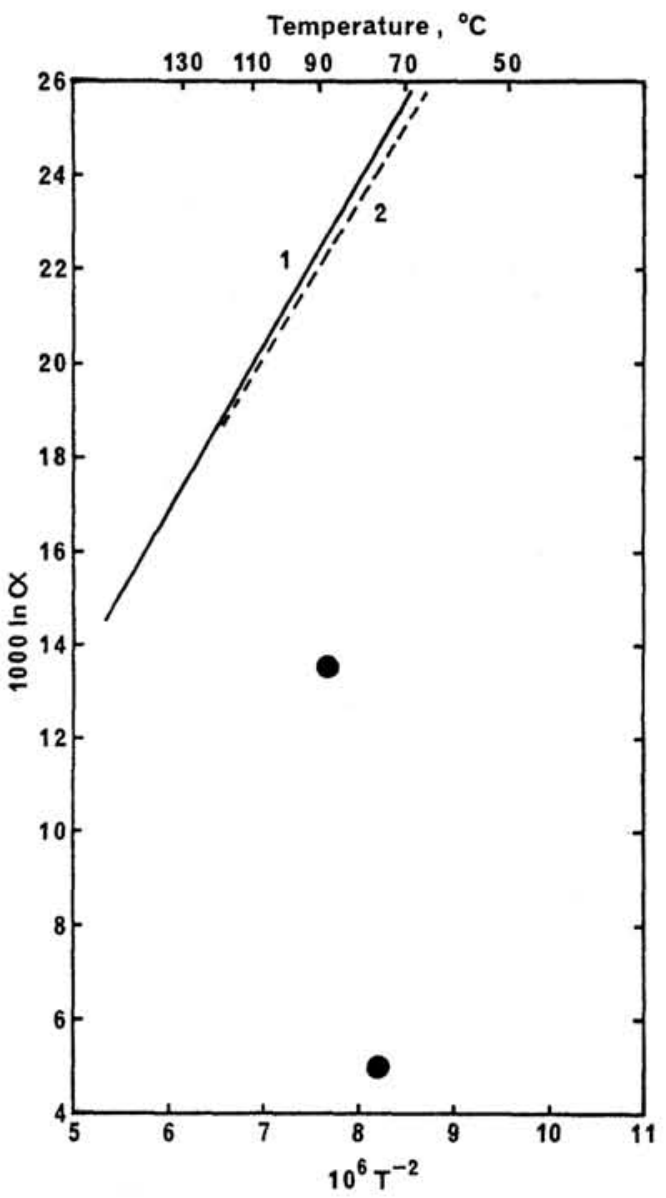

Fig. 2 Comparison of the oxygen isotopic fractionation factors between silica and water obtained in the different conditions of precipitation. Solid circles represent the present results. Line 1, amorphous silica-water system (Kita et al., 1985). Line 2, extrapolation from high temperature experiments 'for the quartz-water system reported by Matsuhisa et al. (1979).

The Otake geothermal water used for this and previous (Kita et al., 1985) experiments contains $525 \mathrm{ppm}$ of silica, in which most of the total silica is ionic. The ionic silica means the silica detected by conventional molybdate yellow method. According to Weres et al. (1981), in aqueous solutions supersaturated with dissolved silica (ionic silica), the excess silica may be converted to colloidal amorphous silica through formation of silica polymers, nucleation of an amorphous silica phase in the form of colloidal particles, and growth of the amorphous silica particles by further chemical deposition of silicic acid on their surfaces. Weres et al. (1981) also suggested that "adsorbed silica" is a reaction intermediate between dissolved silica and solid amorphous silica in the process of growth of amorphous silica particles. The term "adsorbed silica" does not mean adsorption in the usual, physical sense, but implies formation of "loosely bound" silica on the surface of amorphous silica that is rapidly removed from the surface in an alkaline medium. The loosely bound silica is also called "adsorbed silicic acid" (Holt and King, 1955). The adsorbed silica is present on the solid amorphous silica only when the dissolved silica in solution is supersaturated (Weres et al., 1981) and changes to solid amorphous silica through rearrangement and interlinkage on the surface of amorphous silica during the growth. The rearrangement and interlinkage of adsorbed silica is considered to give a key to the mechanism of isotopic variation of the silica precipitation from the geothermal water.

In polymerization of silicic acid, one atom of oxygen must be removed from two silicic acids. This means that the oxygen removing process may be accompanied by a kinetic oxygen isotope effect. The results obtained by the present experiment seem to show the existence of such a kinetic isotope effect. The apparent disequilibrium results, however, may be explained as an equilibrium process by the existence of "adsorbed silica".

In the case of silica precipitated from flowing geothermal water, adsorbed silica existing in the growth of silica on the solid materials changes to amorphous silica through rearrangement and interlinkage as mentioned above. Therefore, the isotopic equilibrium between the silica and hot water can be considered to be attained by the rearrangement and interlinkage of "adsorbed silica". 
Apparently small fractionation factors obtained in the present experiment are probably explained as follows. Polymerization of ionic silica originally contained in the geothermal water must proceed in the solution as it is concentrated (Brown and MacDwell, 1983: Shimono et al., 1983). If the adsorbed silica progressively changes to colloidal silica in isotopic equilibrium with the solution of changing isotopic ratio through rearrangement and interlinkage on the surface of the colloidal silica, the silica must have an isotopic zoning (Fig. 3); lower $\delta^{18} \mathrm{O}$ silica formed at an early stage is encrusted by later formed higher $\delta^{18} \mathrm{O}$ silica. Colloidal silica once formed does not reequilibrate with the solution at later stages. As a consequence, the observed oxygen isotopic ratio of the bulk silica samples should be out of equilibrium with the final solution, and the larger change in the $\delta^{18} \mathrm{O}$ value of solution results in the larger deviation of $\delta^{18} \mathrm{O}$ value from the equilibrium value at the temperature of silica separation (Fig. 3). The isotopic fractionation between bulk silica and solution in the previous experiments (Kita et al., 1985) is almost identical with the equilibrium value as the $\delta^{18} \mathrm{O}$ value of solution is constant through the precipitation. The results obtained in the two different experiments can be explained consistently by the existence of a common reaction intermediate, "adsorbed silica". Therefore, the process of the rearrangement and interlinkage of adsorbed silica on the surface of the silica is inferred to contribute to attaining the oxygen isotopic equilibrium between the silica and water in the precipitation from the geothermal water. When quartz precipitates from a solution, the precipitation may proceed in several steps. That is, the initial formation of "adsorbed silica" is followed by rearrangement and interlinkage, and finally by the formation of quartz. Because there is no significant oxygen isotope fractionation between amorphous silica and quartz (Kita et al., 1985), the main step to determine the oxygen isotope fractionation between quartz and solution would also be the rearrangement and interlinkage of "adsorbed silica".

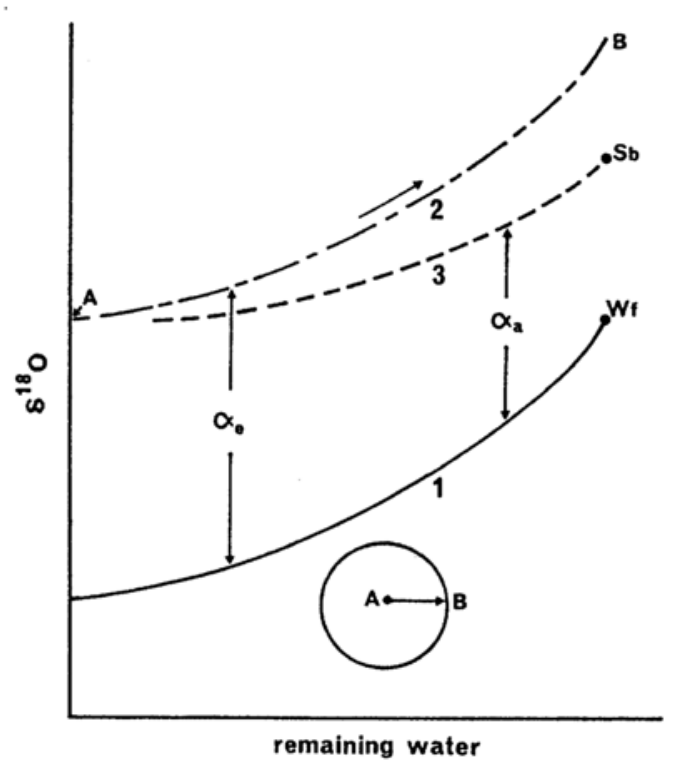

Fig. 3 A schematic diagram showing the growth of a silica particle with isotopic zoning formed by evaporative concentration at constant temperature. Line 1 indicates the gradual increase in $\delta^{18} \mathrm{O}$ value of solution owing to evaporation. Wf corresponds to the solution at the final stage. Line 2 shows the $\delta^{18} O$ variation of amorphous silica phase growing on the surface of a particle in equilibrium with the Line 1 solution. The cross section $A-B$ of the particle shows an outward increase of the $\delta^{18} O$ value. Line 3 represents the cumulative $\delta^{18} O$ values of the silica particle. Sb gives the bulk silica composition when it is separated from the final solution. $\alpha_{e}$ and $\alpha_{a}$ stand for equilibrium and apparent fractionation factors between silica and solution, respectively.

Acknowledgements-We acknowledge Kyushu Electric Power Co. for the use of the reinjection line in Otake Geothermal Power Plant for sampling the geothermal water, and also Dr. O. Matsubaya of Akita University for his encouragement during the course of this work. We thank Dr. M. Kusakabe of Okayama University for comments on the earlier version of the manuscript and Dr. Y. Matsuhisa of Geological Survey of Japan for fruitful discussion. H. Etchu of Akita University helped us in the isotopic analysis of water. This work was supported financially in part by Grant-in-Aid for Encouragement of Young Scientist (No. 59740268) from the Ministry of Education, Science and Culture, Japan. 


\section{REFERENCES}

Brown, K.L. and MacDwell, G.D. (1983) pH control of silica scaling. Proc. 5th NZ Geothermal Workshop 157-161.

Holt, P.F. and King, D.T. (1955) The chemistry of silica surface. J. Chem. Soc. 773-779.

Kita, I. and Matsubaya, O. (1983) $\quad F_{2}-$ technique for the oxygen isotopic analysis of silica minerals. Rep. Res. Inst. Underground Resources, Mining College, Akita Univ. No. 48, 25-34.

Kita, I., Taguchi, S. and Matsubaya, O. (1985) Oxygen isotope fractionation between amorphous silica and water at $34-93^{\circ} \mathrm{C}$. Nature $314,83-84$.

Matsuhisa, Y., Goldsmith, J.R. and Clayton, R.N. (1979) Oxygen isotopic fractionation in the system quartz- albite-anorthite-water. Geochim. Cosmochim. Acta 43, 1131-1140.

O'Neil, J.R. and Epstein, S. (1966) A method for oxygen isotope analysis of milligram quantities of water and some of its applications. J. Geophys. Res. 71, 4955-4961.

Shimono, T., Isobe, T. and Tarutani, T. (1983) Study of the polymerization of silicic acid in aqueous solution by trimethylsilylation-gas chromatography. J. Chromatogr. 258, 73-80.

Weres, O., Yee, A. and Tsao, L. (1981) Kinetics of silica polymerization. J. Colloid Interface Sci. 84, 379-402. 